Check for updates

Cite this: Mater. Adv., 2020, 1,783

Received 17th May 2020

Accepted 9th June 2020

DOI: 10.1039/d0ma00322k

rsc.li/materials-advances

\title{
Efficient ionic medium supported reduced graphene oxide-based sensor for selective sensing of dopamine $\dagger$
}

\begin{abstract}
Nadeem Baig, (D)*abc Abdel-Nasser Kawde (D) *a and Mohamed Ibrahim (D) ${ }^{\mathrm{d}}$
A highly sensitive and electroactive reduced graphene oxide is achieved by directly reducing the ionic medium supported graphene oxide on the electrode surface. The ionic medium supported reduced graphene oxide (im-rGO) has revealed enhanced electrochemical activity compared to water medium reduced graphene oxide under the same set of conditions. The im-rGO has revealed excellent kinetics. The ionic medium supported reduced graphene oxide modified graphite pencil electrode (im-rGO/GPE) was used for selective trace level quantification of dopamine (DA) in human urine. The developed sensor was comprehensively investigated by field emission-scanning electron microscopy (FE-SEM), electrochemical impedance spectroscopy (EIS), cyclic voltammetry (CV), Raman spectroscopy, and Fourier-transform infrared spectroscopy (FTIR). The im-rGO on the electrode surface has effectively increased the electroactive surface area for DA from 0.063 to $0.631 \mathrm{~cm}^{2}$. The charge transfer coefficient $(\alpha)$ and the apparent heterogeneous electron transfer rate constant $\left(k_{\mathrm{s}}\right)$ were calculated to be 0.61 and $5.81 \mathrm{~s}^{-1}$, respectively. A very low LOD of $95 \mathrm{nM}$ was achieved without using any noble or precious metals in combination. The im-rGO/GPE has shown an excellent capability to sense dopamine in the presence of a high concentration of ascorbic acid. The ionic medium supported reduction of GO is the fastest and most cost-effective approach to enhance the sensitivity of the sensor for dopamine in a short time.
\end{abstract}

\section{Introduction}

The graphite pencil electrode (GPE) is a carbon-based renewable electrode. ${ }^{1}$ Graphite pencil lead is a readily available writing tool, and it is extensively used worldwide for writing purposes. The graphite is a major constituent of graphite pencil lead and contributes approximately $65 \%$ in its composition along with about $30 \%$ clay and $5 \%$ binders. $^{2}$ The presence of graphite in the GPE has offered high conductivity to the electrode. Its exploration as an electrode material practically started at the end of the 20th century. ${ }^{3,4}$ It is an emerging

\footnotetext{
${ }^{a}$ Chemistry Department, King Fahd University of Petroleum and Minerals, Dhahran 31261, Saudi Arabia.E-mail: nadeembaig@kfupm.edu.sa, nadeembaig38@gmail.com,akawde@kfupm.edu.sa,ncaug11@gmail.com

${ }^{b}$ Center for Research Excellence in Desalination \& Water Treatment,

King Fahd University of Petroleum and Minerals, Dhahran 31261, Saudi Arabia. E-mail: nadeembaig@kfupm.edu.sa, nadeembaig38@gmail.com

${ }^{c}$ Center for Environment and Water, King Fahd University of Petroleum and Minerals, Dhahran 31261, Saudi Arabia

${ }^{d}$ Department of Clinical Pharmacy Research, Institute for Research and Medical Consultations, Imam Abdulrahman Bin Faisal University, P.O. Box 1982, Dammam 31441, Saudi Arabia

$\dagger$ Electronic supplementary information (ESI) available. See DOI: 10.1039/ d0ma00322k
}

sensing tool, and its electrochemical features are intensively being explored. ${ }^{5}$ The graphite pencil electrode has some obvious advantages over other electrodes. It is cost-effective and readily available. It can work in a wide potential window, with low background current, and exhibits chemical inertness. ${ }^{6}$ Due to the ready availability of the renewable surface of the GPE, the extensive polishing that is required in the case of glassy carbon electrodes can be avoided in GPEs. ${ }^{7}$ It is extruded from the electrode holder to the desired length, and this feature provides control to the exposed area for sensing. ${ }^{8}$ Among the carbon-based electrodes, the GPE, due to its extraordinary features, has received great attention in the field of electrochemical sensors. Various electrodes were evaluated for their sensitivity towards $\alpha$-naphthol, and the graphite pencil electrode has exhibited greater sensitivity over other electrodes. ${ }^{9}$ The graphite pencil electrode has been explored several times for electroanalytical applications. ${ }^{10,11}$

The sensitivity and selectivity of the graphite pencil electrode can be further enhanced by modifying it with various advanced nanostructured materials. ${ }^{12,13}$ Graphite pencil electrode modification is more practical and facile. The graphite pencil electrode is more favorable for the electrodeposition of nanomaterials to improve the surface electroactivity due to its roughness and porosity. Pt-CuO nanocubes were electrodeposited on the 
graphite pencil electrode for non-enzymatic glucose sensing, and a wide linear range was observed. ${ }^{14}$ Kawde et al. ${ }^{15}$ have deposited Au NPs on the surface of the graphite pencil electrode by placing the electrode in a Au NP solution and deposition of the Au NPs took place at $75^{\circ} \mathrm{C}$. The pretreatment of the graphite pencil electrode has also shown a great impact on the sensitivity and selectivity of the electrodes. ${ }^{6,9}$ Through suitable modification of the graphite pencil electrode, the optimum and desired performance of the sensor can be achieved.

Dopamine is a crucial biomolecule and neurotransmitter that performs several functions in the human body. Its abnormal concentration can cause some serious problems in the human body, and its sensing has great biological significance. Dopamine is electrochemically active, and it can be oxidized easily with a suitable electrode material. ${ }^{16,17}$ The electrochemical methods are attractive over other analytical tools due to their simplicity and cost-effectiveness. However, its oxidation potential is close to that of ascorbic acid and uric acid, which strongly overlap the oxidation signal of dopamine. ${ }^{18}$ These potential interferents, specifically ascorbic acid, are present in high concentrations in human fluids that can strongly affect the electrochemical detection of dopamine ${ }^{19}$ Overall, the electrochemical sensors are suffering from surface fouling, selectivity, and suitable sensitivity for dopamine that impede their advancement as a commercial sensor. ${ }^{16}$ The electrode surfaces are modified with several electroactive materials for selective sensing of dopamine. ${ }^{20,21}$ The modified electrodes are still prepared through multistep modification, and generally, noble metal nanoparticles are incorporated to achieve high sensitivity and selectivity. Substantial efforts are being made to introduce smart electroactive materials that can easily incorporate on the electrode surface for selective and sensitive sensing of dopamine.

Graphene has received extraordinary consideration in the advanced material family since its isolation in 2004. It is a two dimensional $\mathrm{sp}^{2}$ hybridized carbon material. ${ }^{22,23}$ Although graphene has a short history, its characteristics in different fields are extensively explored. Due to the unprecedented properties of graphene, it is considered a promising material in the field of biotechnology, electronics, and energy storage. ${ }^{24}$ Graphene has demonstrated excellent conductance, extremely low resistance, and a huge theoretical surface area $\left(2630 \mathrm{~m}^{2} \mathrm{~g}^{-1}\right)$. Graphene possesses perfect electron mobility at room temperature and exceptional mechanical strength. ${ }^{25}$ Graphene is considered an excellent electrode material due to its fast charge transfer, wide potential window, and high electrical conductance. ${ }^{26-29}$ However, graphene is not new for the detection of dopamine. Kim et al. and $\mathrm{X}$. Ma et al. have cast graphene on a GCE, and the estimated detection limit was $2.64 \mu \mathrm{M}$ and $0.5 \mu \mathrm{M}$, respectively. ${ }^{30,31} \mathrm{Y}$. Wang et al. dispersed graphene in CS and dried it overnight on the GCE surface. The limit of quantification was obtained as $5 \mu \mathrm{M} .{ }^{32}$ The graphene nanosheet paste electrode shows the detection limit of $0.6 \mu \mathrm{M} .^{33}$ The 3D reduced graphene oxide-based GCE has reached a $5 \mu \mathrm{M}$ quantification limit and $0.17 \mu \mathrm{M}$ detection limit. ${ }^{34}$ The pristine graphene-based GCE has displayed a detection limit of $2 \mu \mathrm{M}$. In addition to this, various graphene composites were also used to enhance the sensitivity of the electrodes towards dopamine. An overoxidized polypyrrole graphenemodified GCE has a quantification and detection limit of $0.5 \mu \mathrm{M}$ and $0.1 \mu \mathrm{M}$, respectively. ${ }^{35} \mathrm{~S}$. Pruneanu et al. have fabricated $\mathrm{Au} / \mathrm{graphene} \mathrm{AuAg}$ and Au/graphene-Au electrodes for dopamine sensing with a limit of detection $0.205 \mu \mathrm{M}$ and $30.3 \mu \mathrm{M}$, respectively. ${ }^{36} \mathrm{~A}$ graphene- $\mathrm{SnO}_{2}$ nanocomposite based carbon ionic liquid electrode (CILE) has a detection limit of $0.13 \mu \mathrm{M} .{ }^{37}$ Moreover, many graphene-based electrodes like PAM/ rGO modified GCE, ${ }^{38} \mathrm{RGO} / \beta$-CD-pyrrole GCE, ${ }^{39} \mathrm{MgO} /$ graphene/ tantalum electrode,${ }^{40}$ and $\mathrm{mp}-\mathrm{GR} / \mathrm{GCE}^{41}$ were also used for the detection of dopamine. However, still, there is a need to develop an efficient reduction of graphene oxide on the electrode surface through a facile and cost-effective route to achieve high selectivity and sensitivity for dopamine.

In this work, im-rGO/GPE is introduced for the trace level quantification of dopamine. It has been observed that im-rGO/ GPE is much more sensitive for dopamine compared to the previously reported electrodes in which the graphene was the only modifier. The im-rGO/GPE possesses better or comparable sensitivity to the graphene composite electrodes that were fabricated through complex, tedious, and time-consuming routes. Ionic medium supported-graphene oxide offered a direct reduction of graphene oxide in a short time that imparted high sensitivity to the electrode compared to water medium dispersed graphene oxide. The charge transfer capability of the im-rGO/GPE was significantly enhanced, and well defined sharp reversible peaks of the analytes were observed compared to the response of $\mathrm{w}-\mathrm{rGO} / \mathrm{GPE}$. The developed method of electrode modification is fast, simple, and easy to apply for practical applications. The developed sensor has displayed an excellent capability to detect dopamine in the presence of a high concentration of ascorbic acid. The specificity for dopamine may be attributed to some residual oxygen-containing moieties on the modified sensor. The developed electrode was evaluated by advanced characterization and through its detailed electrochemical study for dopamine sensing.

\section{Experimental}

\subsection{Reagents}

Phenylalanine and alanine were purchased from Fluka (United States of America). Dopamine, L-tyrosine, fructose, glucose, ascorbic acid, L-methionine, uric acid, potassium chloride, and sodium chloride were received from Sigma-Aldrich (United States of America). Graphite was obtained from Fischer Science Education (United States of America). Di-potassium hydrogen orthophosphate and sodium phosphate monobasic were purchased from BDH (England). Double-distilled water was used for all reagent preparations, and it was collected from a Water Still Aquatron A 4000D (England), a lab established unit.

\subsection{Instrumentation and apparatus}

The electrochemical workstation (Auto-Lab, Netherland) was used for all electrochemical measurements. The three-electrode system was used for the measurements. It consisted of $\mathrm{Ag} / \mathrm{AgCl}$ 
as a reference electrode, platinum as a counter electrode, and the graphite pencil as the working electrode. The weight and the $\mathrm{pH}$ control were carried out by a GR-2000 and Accumet ${ }^{\mathbb{R}}$ XL50 pH meter, respectively. The FTIR and Raman spectra were recorded by a NICOLET 6700 FT-IR and HORIBA Scientific LabRAM HR Evolution, respectively. The FE-SEM images of various pencil electrodes were collected by a TESCAN LYRA 3 instrument.

\subsection{Synthesis of graphene oxide}

Graphene oxide was synthesized by slight changes in the famous Hummers' method. ${ }^{42}$ In brief, $2 \mathrm{~g}$ of graphite powder was dispersed in a beaker containing $6 \mathrm{~mL} \mathrm{HNO}_{3}$ and $140 \mathrm{~mL}$ $\mathrm{H}_{2} \mathrm{SO}_{4}$ in an ice bath, and the temperature was controlled below $5{ }^{\circ} \mathrm{C}$. The mixture was kept under stirring in an ice bath for a certain time. After that, $10 \mathrm{~g} \mathrm{KMnO}_{4}$ was slowly added into the mixture. After the addition of $\mathrm{KMnO}_{4}$, the temperature of the mixture was increased to $35{ }^{\circ} \mathrm{C}$ and kept stirring for another 4 hours. After 4 hours, about $240 \mathrm{~mL}$ of double distilled water was slowly added into the mixture, and the temperature should remain below $50{ }^{\circ} \mathrm{C}$. This process should be performed carefully as a lot of heat was released during the dilution of highly concentrated acids. In the next step, the temp was increased to $95{ }^{\circ} \mathrm{C}$ for a certain time. At this point, the mixture appeared brown, which was an indication of the formation of the graphene oxide. After this, the reduction of the remaining oxidant was carried out by the dropwise addition of $15 \mathrm{~mL}$ of $30 \% \mathrm{H}_{2} \mathrm{O}_{2}$ into the mixture. In the final stage, the extensive washing of the graphene oxide mixture was carried out with the help of $\mathrm{HCl}$ and double distilled water to bring the $\mathrm{pH}$ to about 6.5. After thorough cleaning, the graphene oxide dispersion was dried and stored for further experimental study.

\subsection{Electrode modification procedure}

The dispersion of graphene oxide was prepared in different ionic media. A $2 \mathrm{mg} \mathrm{mL}^{-1}$ graphene oxide solution was produced in various media such as double-distilled water (w-GO), $0.1 \mathrm{M} \mathrm{KCl}$ (pc-GO), 0.1 M PB (pb-GO) and 0.1 M acetate buffer (ac-GO). The $7 \mathrm{~mm}$ of graphite pencil electrode extruded from the holder was dipped into the various graphene oxide dispersed media along with the $\mathrm{Ag} / \mathrm{AgCl}$ reference electrode and platinum wire as a counter electrode for the study of the ionic medium effect. The graphene oxide from the respective medium was reduced on the surface of the GPE by sweeping the potential in the range of -1.4 to $0.3 \mathrm{~V}$ at the scan rate of $0.02 \mathrm{~V} \mathrm{~s}^{-1}$ for two cycles. The graphene oxide concentration in the best ionic medium was optimized by preparing the dispersion in the range of 1 to $10 \mathrm{mg} \mathrm{mL}^{-1}$ in $0.1 \mathrm{M}$ acetate buffer. The study of dopamine was performed under optimum electrode modification conditions. The optimum conditions were found as $4 \mathrm{mg} \mathrm{mL} \mathrm{m}^{-1}$ of ac-GO, two reduction cycles, -1.4 to $0.3 \mathrm{~V}$ reduction potential window, and $0.02 \mathrm{~V} \mathrm{~s}^{-1}$ scan rate. The graphite pencil electrode each time was modified under the aforementioned set of conditions. The same geometrical area of the graphite pencil electrode was modified and used throughout the electrochemical optimization and electrochemical analysis. The geometrical area of the GPE used for analysis consisted of $11.39 \mathrm{~mm}^{2}$. After each measurement, the electrode modified surface was renewed to eliminate the byproduct effect of dopamine. After modification, the electrode surface was washed gently by dipping twice in double-distilled water before analysis.

\subsection{Real sample analysis}

A human urine sample was collected from a healthy individual in a plastic bottle. The collected urine sample was further diluted to 200 folds with $0.1 \mathrm{M}$ phosphate buffered saline before analysis. Apart from dilution, no further treatment of the urine sample was performed. Various known concentrations of dopamine were spiked into the urine sample, and their recoveries were found out.

\section{Results and discussion}

\subsection{Raman and IR spectra of GO}

In Fig. S1A (ESI $\dagger$ ) the Raman spectra of the graphite and synthesized graphene oxide can be seen. Graphene oxide was synthesized by using the famous Hummers' method. ${ }^{42}$ The well-resolved characteristic D, G, and 2D band appeared in the graphene oxide Raman spectrum. The $\mathrm{D}$ band in the Raman spectrum of the graphene oxide appeared due to the defects in the graphene oxide. The $\mathrm{G}$ band indicates the $\mathrm{E}_{2 \mathrm{~g}}$ phonons of the $\mathrm{sp}^{2}$ planner configuration present in the graphene oxide. The $2 \mathrm{D}$ band is considered the overtone of the $\mathrm{D}$ band. A weak $\mathrm{D}$ band appeared in the Raman spectrum of graphite. The strong $\mathrm{D}$ band in the graphene oxide indicated that the extensive oxidation of the graphite had produced defects in the $\mathrm{sp}^{2}$ planar structure of the graphite. The FTIR spectra of the graphite before oxidation and after oxidation have been scanned. A prominent IR absorption peak has been observed in the case of graphene oxide (Fig. S1Bb, ESI $\dagger$ ) compared to the graphite (Fig. S1Ba, ESI $\dagger$ ). The broad peak of $-\mathrm{OH}$ is observed from $2850 \mathrm{~cm}^{-1}$ to $3741 \mathrm{~cm}^{-1}$. The FTIR peak that appeared in the range of $1698 \mathrm{~cm}^{-1}$ to $1794 \mathrm{~cm}^{-1}$ represented the carbonyl functional group, and the $\mathrm{C}=\mathrm{C}$ peak could be observed from $1531 \mathrm{~cm}^{-1}$ to $1670 \mathrm{~cm}^{-1}$. The $\mathrm{C}=\mathrm{C}$ weak peak is also observed in the graphite FTIR spectrum. The $\mathrm{C}-\mathrm{O}$ stretching peaks due to alkoxy, epoxy and carboxylic acid functional groups have appeared from $906 \mathrm{~cm}^{-1}$ to $1496 \mathrm{~cm}^{-1} .43$

\subsection{Ionic medium supported graphene oxide reduction on GPE (im-rGO/GPE)}

The graphite pencil electrode elongated shape, and surface roughness provided a better opportunity for direct electrochemical reduction of graphene oxide. For the fabrication of a single-use electrode surface, the modification process must be fast and should be achieved in a short time. GO is easily dispersed in water due to the presence of polar functional groups. It is observed that the small number of CV reduction cycles of the water medium dispersed GO were not enough to enhance the sensitivity of the GPE to the optimum level. The ionic medium supported graphene oxide has shown a dramatic effect on the 
sensitivity of the sensor. The sensitivity of the various GPE surfaces was analyzed using $2 \mathrm{mM} \mathrm{K}_{3} \mathrm{Fe}(\mathrm{CN})_{6} / \mathrm{K}_{4} \mathrm{Fe}(\mathrm{CN})_{6}$ in $0.1 \mathrm{M} \mathrm{KCl}$ solution. The effect of various ionic media was investigated by a reduction of $2 \mathrm{mg} \mathrm{mL}^{-1} \mathrm{GO}$ on the GPE surface. The electrochemical reduction was performed by scanning of $\mathrm{CV}$ from -1.4 to $0.3 \mathrm{~V}$ over 2 cycles. The study of the CV behavior of the reduced graphene oxide on GPE in the presence of ionic medium has shown a great impact on the peak current and peak shape (Fig. S2, ESI $\dagger$ ). The peak current and reversibility of $\mathrm{K}_{3} \mathrm{Fe}(\mathrm{CN})_{6} / \mathrm{K}_{4} \mathrm{Fe}(\mathrm{CN})_{6}$ were significantly improved using im-rGO/GPE compared to w-rGO/GPE. The electroactive surface area study of the GPE was performed after reducing the water medium dispersed graphene oxide and various ionic medium dispersed graphene oxide, such as in $0.1 \mathrm{M} \mathrm{KCl}$ (pc-GO), 0.1 M PB (pb-GO) and 0.1 M acetate buffer (ac-GO). The responses of various rGO modified electrodes were recorded by scanning the CVs at different scan rates of 20 to $100 \mathrm{mV} \mathrm{s}^{-1}$ in $0.1 \mathrm{M} \mathrm{KCl}$ containing $2 \mathrm{mM} \mathrm{K}_{3} \mathrm{Fe}(\mathrm{CN})_{6} /$ $\mathrm{K}_{4} \mathrm{Fe}(\mathrm{CN})_{6}$. The electroactive surface area was calculated to be about $0.112,0.185,0.219$ and $0.246 \mathrm{~cm}^{2}$ for w-rGO/GPE, pc-rGO/ GPE, pb-rGO/GPE and ac-rGO/GPE, respectively. The surface area study revealed that the ionic medium supported GO (im-GO) reduction on the graphite pencil electrode provided a better electroactive surface area. The direct electrochemical reduction of graphene oxide in the ionic medium is attractive and valuable to attain the required sensor sensitivity in a short time. An acetate medium has shown more effective results compared to other media. For further study, ac-GO was used for the modification of the GPE (Fig. S2, ESI $\dagger$ ).

\subsection{Optimization of im-GO}

The synthesized graphene oxide was dried in open-air. The dried graphene oxide was dispersed in $0.1 \mathrm{M}$ acetate buffer and sonicated for 2 hours to get a uniform and stabilized dispersion. Various concentrations of graphene oxide solution were prepared in the range of $1-10 \mathrm{mg} \mathrm{mL}^{-1}$ to find the best response of the concentration of graphene oxide in the ionic medium that could be effectively reduced on the GPE surface. The best response of the modified sensor was obtained when the concentration of the GO was $4 \mathrm{mg} \mathrm{mL} \mathrm{m}^{-1}$ in the ionic medium. The number of $\mathrm{CV}$ reduction cycles was optimized from 1 to 6 . The maximum response was observed at two cycles, and the effective scan window for graphene oxide reduction was found to be -1.4 to $0.3 \mathrm{~V}$. The scan rate for GO electrochemical reduction was optimized from 0.005 to $0.05 \mathrm{~V} \mathrm{~s}^{-1}$, the current was enhanced until $0.02 \mathrm{~V} \mathrm{~s}^{-1}$ and decreased gradually as the scan rate was further increased. All these parameters were optimized for a $1 \mathrm{mM}$ concentration of dopamine. Various electrolytes were also scanned for $1 \mathrm{mM}$ dopamine, and the most suitable one was found to be PBS due to the sharp and high peak current for dopamine. All of the studies were completed by modifying the GPE in the potential window of -1.4 to $0.3 \mathrm{~V}$ at a scan rate of $0.02 \mathrm{~V} \mathrm{~s}^{-1}$ for two cycles. The reduction current near $-1.4 \mathrm{~V}$ was sharply increased in the 2 nd cycle. This was an indication that graphene oxide was reduced during the first cycle, and its reduction was continued in the 2nd cycle (Fig. 1). Different voltammetric

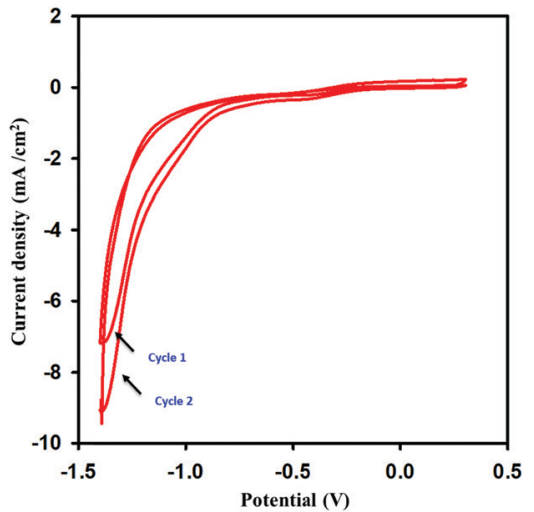

Fig. 1 Reduction of graphene oxide by scanning the $C V$ in the range of -1.4 to $0.3 \mathrm{~V}$ at a scan rate of $0.02 \mathrm{~V} \mathrm{~s}^{-1}$ over 2 cycles.

techniques like CV, DPV, LSV, and SWV were examined, and the SWV was found to be more effective for dopamine sensing.

\subsection{Surface characterization of the bare GPE and im-rGO/GPE}

Scanning electron microscopy could assist in revealing the presence of im-rGO on the surface of the GPE. The detailed morphology of the bare and the im-rGO/GPE was investigated at low and high magnification. The images scanned at $1 \mu \mathrm{m}$ revealed the presence of a wrinkled graphene sheet on the surface of the GPE. These wrinkled graphene sheets became clearer at higher magnification of $500 \mathrm{~nm}$ (Fig. 2Ba and $\mathrm{Bb}$ ). However, on the bare GPE no such layers or wrinkles were observed (Fig. 2Aa and Ab). The FE-SEM images are evidence that graphene successfully formed on the surface of the im-rGO/GPE.

A
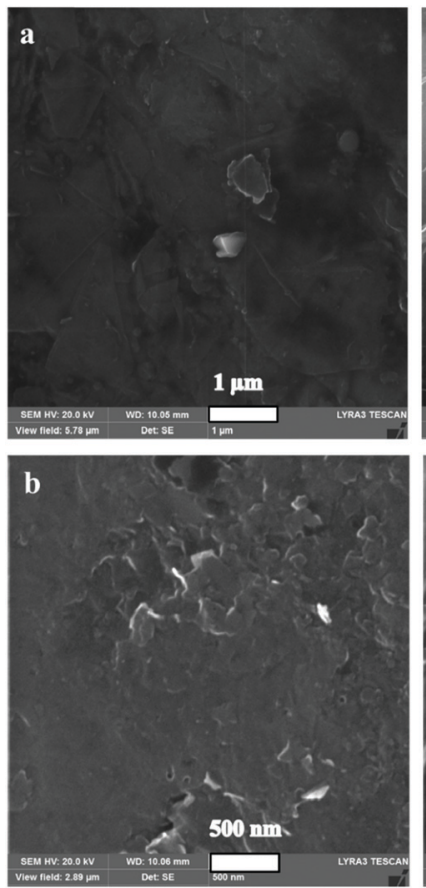

Fig. 2 SEM images of (A) bare GPE and (B) im-rGO/GPE at low and high magnification of (a) $1 \mu \mathrm{m}$, and (b) $500 \mathrm{~nm}$. 
The graphene wrinkles on the electrode surface area further contribute to improving the active surface area and providing active sites for the electrochemical reaction. These wrinkles are also helpful in preventing the restacking of the reduced graphene oxide. ${ }^{44}$ The reduced graphene oxide presence on the surface of the GPE could be further confirmed by the EIS investigation. The reduced graphene oxide is an excellent conductor and exhibits high charge transfer. Its presence on the electrode surface helps to overcome the charge transfer resistance. In this regard, the electrochemical behavior of the bare and the modified electrode was examined by EIS. The EIS characterization was performed in $0.1 \mathrm{M} \mathrm{KCl}$ solution comprising $5 \mathrm{mM} \mathrm{K}_{3} \mathrm{Fe}(\mathrm{CN})_{6} / \mathrm{K}_{4} \mathrm{Fe}(\mathrm{CN})_{6}$, and the frequency range was scanned from $0.01 \mathrm{~Hz}$ to $100 \mathrm{kHz}$. The Nyquist plots consist of two portions. One is linear, whereas the other part consists of a semicircle. The semicircle part that appeared at a higher frequency is directly related to the electron transfer limited process. The linear part at a lower frequency corresponds to the diffusion control process. The EIS spectra have revealed the extensive change in the behavior of the GPE surface after modification with im-rGO. The charge transfer resistance $\left(R_{\mathrm{CT}}\right)$ value calculated from the semicircle of the Nyquist plot of the bare GPE was about $2746 \Omega$ (Fig. 3Ab). The EIS spectra of im-rGO/GPE have almost demonstrated a straight line, and it was difficult to observe the presence of a semicircle (Fig. 3Aa). The charge transfer resistance values of the bare, and the modified electrode indicated that the resistance was substantially reduced by the graphene layer on the electrode. The same phenomena were also demonstrated by the Bode phase plots (Fig. 3B). At higher frequencies, the bare GPE (Fig. 3Bb) has a Bode phase angle $62.5^{\circ}$, and the im-rGO/GPE (Fig. 3Ba) phase peak disappeared at higher frequencies. This revealed the high charge transfer of the modified surface due to its low charge transfer resistance. ${ }^{45}$ The EIS study revealed that the charge transfer resistance of the GPE has considerably reduced after the formation of the im-rGO layer on it. The electrochemical behavior of the bare and im-rGO/GPE towards dopamine was further examined by CV (Fig. 3C). The CVs were obtained for $1 \mathrm{mM}$ dopamine with bare GPE and im-rGO/GPE in PBS buffer
(0.1 M, pH 6.8). On the bare electrode surface, a broad peak of dopamine was observed and produced current because the electrochemical reaction of dopamine was small (Fig. 3Cb). The electrochemical response of the dopamine on im-rGO/GPE was entirely different. The oxidation and reduction peak sharpness of dopamine was substantially improved. This is evident from the fact that the electroactivity of the GPE for dopamine was substantially improved after the formation of the im-rGO layer (Fig. 3Ca).

\subsection{Study of the scan rate effect and electroactive surface area for dopamine}

The electrode kinetics for dopamine were further explored by cyclic voltammetry. The effect of the scan rate on dopamine was examined by cyclic voltammetry. The scan rate variation of $\mathrm{CV}$ has shown a significant effect on the peak current of the dopamine. On the modified electrode surface, the current increased linearly as the scan rate increased for $1 \mathrm{mM}$ dopamine (Fig. 4A). The same effect has been observed for the bare electrodes (Fig. 4B). However, at a higher scan rate, the current response was dramatically decreased on the bare electrode surface. At a higher scan rate, the short time is available for the completion of the electrochemical reaction on the electrode surface; the bare electrode may not support the fast charge transfer due to its poor electro-kinetic. The modified electrode responded appropriately and efficiently at a higher scan rate for dopamine electrochemical reactions. The Randles-Sevcik equation (eqn (1) $)^{46}$ was used for the calculation of the electroactive surface area for bare and im-rGO/GPE. The im-rGO/GPE electrode was developed under the standard and optimized set of conditions for the measurement of the electroactive surface area.

$$
I_{\mathrm{p}}=2.69 \times 10^{5} \nu^{1 / 2} n^{3 / 2} C D^{1 / 2} A,
$$

where $I_{\mathrm{p}}$ is the peak current $(\mathrm{A}), \nu$ is the scan rate $\left(\mathrm{V} \mathrm{s}^{-1}\right)$, $n$ is the number of electrons, $C$ is the analyte concentration ( $\left.\mathrm{mol} \mathrm{L} \mathrm{L}^{-1}\right), D$ is the diffusion coefficient $\left(\mathrm{cm}^{2} \mathrm{~s}^{-1}\right)$, and $A$ is the electrode electroactive surface area $\left(\mathrm{cm}^{2}\right)$. The electroactive surface area was calculated by using bare GPE or im-rGO/GPE by scanning the different CVs at a scan rate from the range of $20 \mathrm{mV} \mathrm{s}^{-1}$ to $120 \mathrm{mV} \mathrm{s}^{-1}$ from a solution containing $5 \mathrm{mM}$
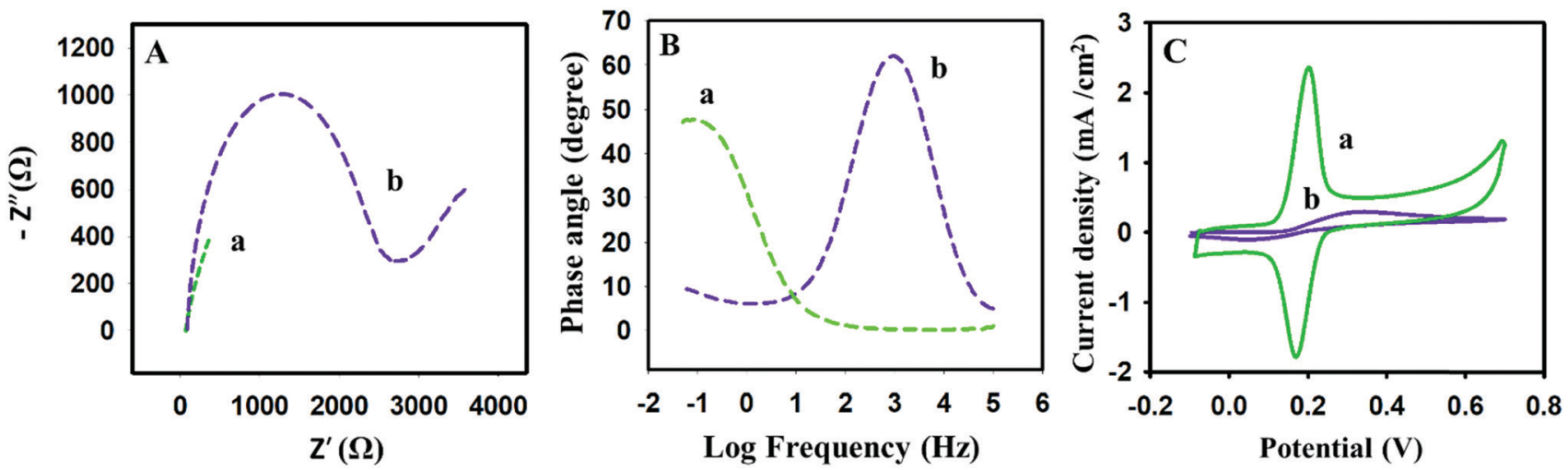

Fig. 3 (A) Nyquist and (B) Bode phase plots of $5 \mathrm{mM} \mathrm{K}{ }_{3} \mathrm{Fe}(\mathrm{CN})_{6} / \mathrm{K}_{4} \mathrm{Fe}(\mathrm{CN})_{6}$ in $0.1 \mathrm{M} \mathrm{KCl}$ solution on (a) im-rGO/GPE, and (b) the bare GPE upon application of a frequency range from $0.01 \mathrm{~Hz}$ to $100 \mathrm{kHz}$. (C) $\mathrm{CVs}$ of (a) im-rGO and (b) the bare GPE were recorded at $0.1 \mathrm{~V} \mathrm{~s}{ }^{-1}$ in a $1 \mathrm{mM}$ dopamine 0.1 M PBS buffer (pH 6.8). 

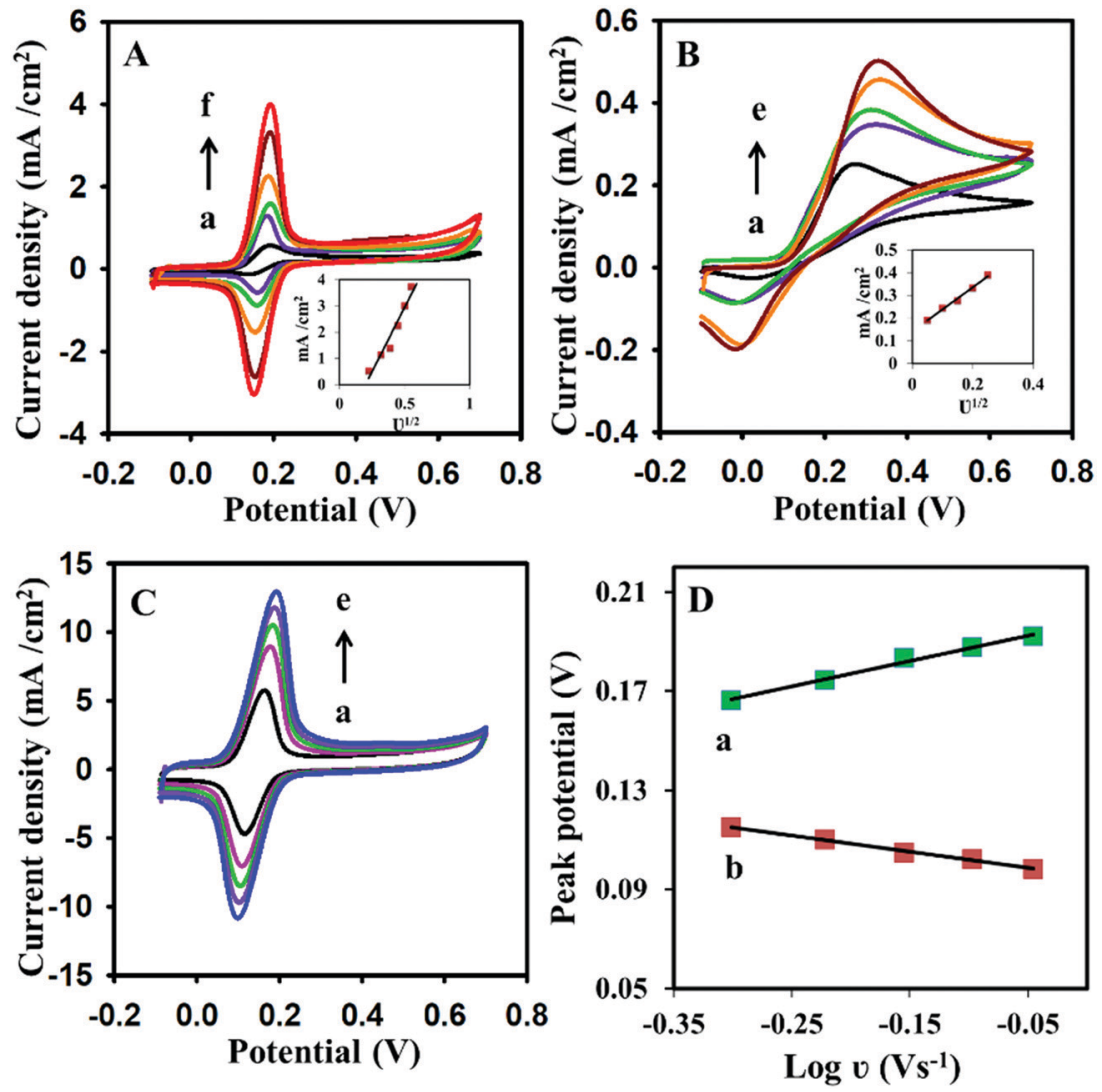

Fig. 4 (A) Cyclic voltammograms of $1 \mathrm{mM}$ dopamine in PBS buffer (0.1 M, pH 6.8) using (A) im-rGO/GPE at scan rates of (a) 50, (b) 100, (c) 150, (d) 200, (e) 250 , and (f) $300 \mathrm{mV} \mathrm{s}^{-1}$; or using (B) the bare GPE at scan rates of (a) 50, (b) 100, (c) 150, (d) 200, and (e) $250 \mathrm{mV} \mathrm{s}^{-1}$. (C) The cyclic voltammograms of $1 \mathrm{mM}$ dopamine using im-rGO/GPE at a higher scan rate (a) 500, (b) 600, (c) 700, (d) 800 , and (e) $900 \mathrm{mV} \mathrm{s}^{-1}$. (D) The linear relationship between log $\nu$ vs. (a) anodic and (b) cathodic peak potential of the cyclic voltammograms at 500 to $900 \mathrm{mV} \mathrm{s}^{-1}$. The insets in (A) and (B) show the linear relationship between current and the square root of the scan rates $\left(\nu^{1 / 2}\right)$.

$\mathrm{K}_{3} \mathrm{Fe}(\mathrm{CN})_{6} / \mathrm{K}_{4} \mathrm{Fe}(\mathrm{CN})_{6}$ in $0.1 \mathrm{M} \mathrm{KCl}$ solution. The electroactive surface area calculated for the bare GPE and the im-rGO/GPE was $0.0628 \mathrm{~cm}^{2}$ and $0.372 \mathrm{~cm}^{2}$, respectively (data not shown). The electroactive surface area calculated for $1 \mathrm{mM}$ dopamine in $0.1 \mathrm{M}$ PBS buffer with bare GPE and im-rGO/GPE was $0.063 \mathrm{~cm}^{2}$ and $0.631 \mathrm{~cm}^{2}$, respectively. The value of the dopamine diffusion coefficient $5.40 \times 10^{-6} \mathrm{~cm}^{2} \mathrm{~s}^{-1}$ was used. ${ }^{47}$ The huge surface area for dopamine compared to $\mathrm{K}_{3} \mathrm{Fe}(\mathrm{CN})_{6} / \mathrm{K}_{4} \mathrm{Fe}(\mathrm{CN})_{6}$ indicated that the im-rGO on the electrode surface has shown an affinity towards dopamine. However, the bare electrode surface area for dopamine was close to $\mathrm{K}_{3} \mathrm{Fe}(\mathrm{CN})_{6} / \mathrm{K}_{4} \mathrm{Fe}(\mathrm{CN})_{6}$. The possible affinity of the im$\mathrm{rGO} / \mathrm{GPE}$ for dopamine might be due to the presence of residual functional groups after the reduction of graphene oxide. After the reduction of graphene oxide, the limited number of carboxyl groups was still retained by the surface. ${ }^{48}$ These residual oxygen-containing functionalities such as carboxyl groups provided additional affinity along with the huge surface area of the reduced graphene oxide for dopamine, and this behavior could contribute to substantially improving the sensor response for dopamine (Scheme 1).

The electrode surface plays a key role in controlling the kinetics and the reversibility of the electrochemical reaction. ${ }^{49}$ The information on the reversibility of the electrochemical reaction could be obtained from the cyclic voltammetry. The potential difference

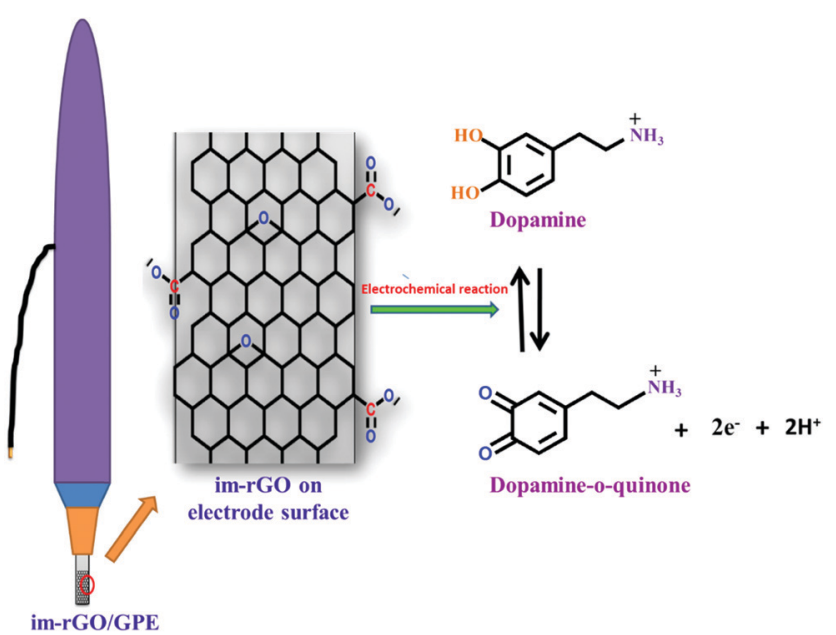

Scheme 1 Electrochemical reaction of dopamine on the surface of the im-rGO/GPE.

between the anodic peak potential and the cathodic peak potential of the reversible reaction is presented in eqn (2).

$$
\Delta E=E_{\mathrm{pa}}-E_{\mathrm{pc}}=59 / n
$$


where $E_{\mathrm{pa}}$ is anodic peak potential $(\mathrm{mV}), E_{\mathrm{pc}}$ is cathodic peak potential $(\mathrm{mV})$, and $n$ is the number of electrons. The value of $\Delta E$ for the reversible reaction is $59 / n \mathrm{mV}$, while for a quasireversible reaction it is greater than $59 / n$, and the only single peak appeared in the case of an irreversible reaction. The $\Delta E$ calculated from CV (Fig. 3Ca) on the modified surface for dopamine was $33.8 \mathrm{mV}$. The value was close to $2.3 R T / n F$ or $59 / n \mathrm{mV}$, which indicated that the electrochemical reaction of dopamine on the modified surface was quasi reversible. The value of $n$ calculated from eqn (2) was 1.745 . This value suggested that two electrons were involved in the electrochemical reaction of the dopamine on the im-rGO/GPE.

The surface coverage $(\Gamma)^{50}$ for dopamine on the electrode surface was calculated by using eqn (3).

$$
\Gamma=I_{\mathrm{p}} 4 R T / n^{2} F^{2} A \nu
$$

where $I_{\mathrm{p}}$ is the peak current, $A$ is the surface area, and $\nu$ is the scan rate. Eqn (3) showed that surface coverage has a direct relation with peak current. The surface coverage calculated for the bare and modified GPE for $1 \mathrm{mM}$ dopamine in 0.1 M PBS buffer was $4.721 \times 10^{-14}$ and $6.179 \times 10^{-10} \mathrm{~mol} \mathrm{~cm}^{-2}$, respectively. This high surface coverage of the modified GPE can be attributed to the presence of the im-rGO on the electrode surface.

In addition to this, at a higher scan rate from 500 to $900 \mathrm{mV} \mathrm{s}^{-1}$ a linear relationship has been observed between the logarithm of the scan rate $(\log \nu)$ and peak potential of the dopamine (Fig. 4). The peak shift was observed for both oxidation and the reduction peak potential. The positive peak potential shift for the oxidation and negative peak potential shift for the reduction of the dopamine was observed on the modified electrode surface (Fig. 4D). For anodic peak potential $\left(E_{\mathrm{pa}}\right)$ and cathodic peak potential $\left(E_{\mathrm{pc}}\right)$ shift, two straight-line equations (eqn (4) and (5)) were yielded.

$$
\begin{gathered}
E_{\mathrm{pa}}(\mathrm{V})=0.1033 \log \nu+0.1977\left(R^{2}=0.9919\right) \\
E_{\mathrm{pc}}(\mathrm{V})=-0.0652 \log \nu+0.0953\left(R^{2}=0.9956\right)
\end{gathered}
$$

According to Laviron's theory, ${ }^{51}$ the slopes of the anodic and cathodic peak potential $v s$. $\log \nu$ are $2.3 R T /(1-\alpha) n F$ and $-2.3 R T / \alpha n F$, respectively. The value of the charge transfer coefficient $(\alpha)$ was calculated as 0.61 using the following eqn (6).

$$
\log k_{\mathrm{a}} / k_{\mathrm{c}}=\log \alpha / 1-\alpha
$$

Moreover, the apparent heterogeneous electron transfer rate constant $\left(k_{\mathrm{s}}\right)$ on the single-use im-rGO/GPE was calculated by using another Laviron's eqn (7) for a surface controlled transfer model: ${ }^{51}$

$$
\begin{gathered}
\log k_{\mathrm{s}}=\alpha \log (1-\alpha)+(1-\alpha) \log \alpha-\log (R T / n F \nu) \\
-\alpha(1-\alpha) n F \Delta E_{\mathrm{p}} / 2.3 R T
\end{gathered}
$$

where $R, T, F, \nu$ and $\Delta E_{\mathrm{p}}$ are gas constant, temperature, Faraday constant, scan rate, and the peak potential separation of the redox pair. In the equation, $n$ is the number of electrons. The value of $n$ for dopamine is 2 (eqn (2)). The value of $k_{\mathrm{s}}$ calculated by using eqn (7) was $5.81 \mathrm{~s}^{-1}$. The $k_{\mathrm{s}}$ value is larger compared to

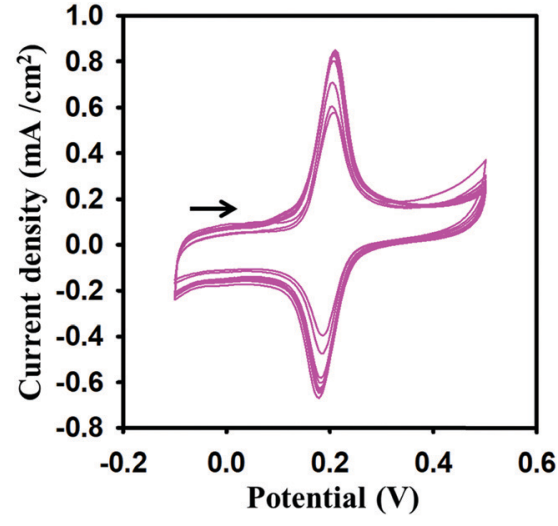

Fig. 5 Multiple cyclic voltammograms at $0.1 \mathrm{~V} \mathrm{~s}^{-1}$ scan rate on the im-rGO/ GPE surface for $0.2 \mathrm{mM}$ dopamine in $0.1 \mathrm{M}$ PBS buffer $(\mathrm{pH} 6.8$ ).

reported values of $0.25 \mathrm{~s}^{-151}$ and $0.77 \mathrm{~s}^{-152}$ for dopamine. The kinetics study has endorsed that the presence of im-rGO on the electrode surface has facilitated the fast charge transfer and favored the fast-electrochemical reaction of dopamine.

The presence of ionic medium supported graphene has not only enhanced the electrochemical performance of the GPE but also shown attraction for dopamine. This was evaluated by the multiple cyclic voltammograms scanned for $0.2 \mathrm{mM}$ dopamine solution in 0.1 M PBS buffer (Fig. 5). The enhancement of the current was observed as the CV scan moved from the one cycle to the next cycle. The sharp current increment in the start indicated that the im-rGO layer on the GPE has a good affinity for dopamine, and it was adsorbed on the electrode surface. This phenomenon was helpful for trace level quantification of dopamine. After continuous multiple cycles, the current increase has become negligible, possibly due to surface saturation or the effect of the byproducts produced on the electrode surface.

\subsection{Effect of $\mathbf{p H}$}

The $\mathrm{pH}$ effect on dopamine was probed from 5.0 to $8.0 \mathrm{pH}$ for $50 \mu \mathrm{M}$ dopamine in $0.1 \mathrm{M}$ PBS buffer. The $\mathrm{pH}$ of the supporting electrolyte has shown a great impact on the current and oxidation peak potential of dopamine. The current was improved as the $\mathrm{pH}$ increased, and the maximum current was obtained at pH 6.5 (Fig. 6). The current was decreased with a further increase in $\mathrm{pH}$. The $\mathrm{pH}$ also displayed a significant effect on the peak potential of dopamine. The peak potential was shifted from $298 \mathrm{mV}$ to $122 \mathrm{mV}$ as the $\mathrm{pH}$ increased from 5.0 to 8.0. The peak potential was moved to negative potential with the increment of sensing medium $\mathrm{pH}$. A linear relationship was observed between peak potential and $\mathrm{pH}$ (Fig. 6B inset). The regression constant $\left(R^{2}\right)$ obtained from the straight-line equation 8 was 0.9994 . The slope was $-58.6 \mathrm{mV}$ per $\mathrm{pH}$ unit, and it was almost equal to the theoretical value of $-59 \mathrm{mV}$ per $\mathrm{pH}$ unit.

$$
E \text { vs. } \mathrm{Ag} / \mathrm{AgCl}=58.97-58.6[\mathrm{pH}]
$$

The slope of eqn (8) describes that equal numbers of electrons and protons are involved in the electrochemical reaction of dopamine on the im-rGO/GPE surface. The number of electrons obtained from eqn (2) was 2 . It has been confirmed 

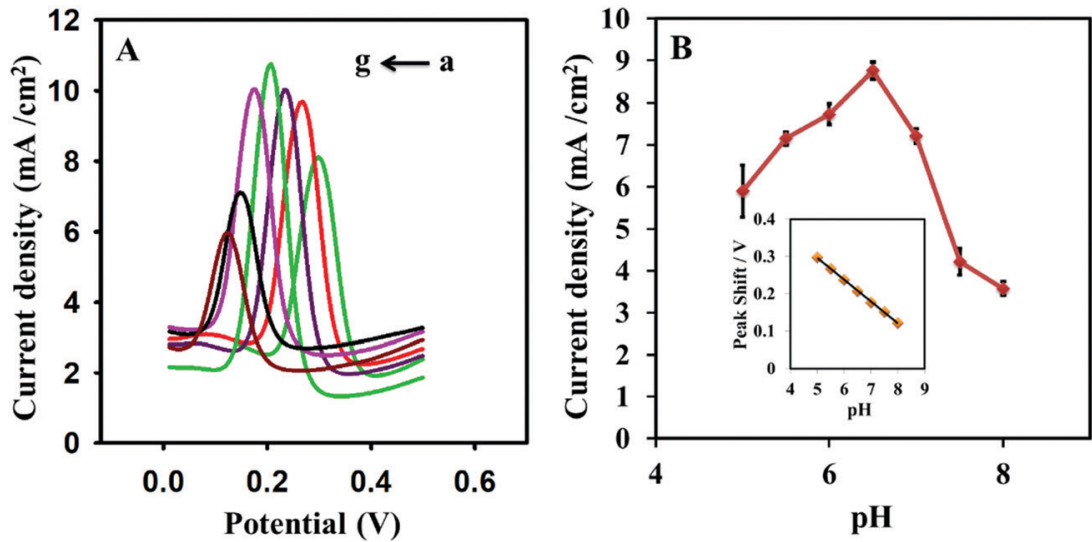

Fig. 6 (A) Square wave voltammograms in 0.1 M PBS solution containing $50 \mu \mathrm{M}$ dopamine at various pH values (a) 5.0, (b) 5.5, (c) 6.0, (d) 6.5, (e) 7.0, (f) 7.5, and (g) $8.0 \mathrm{pH}$ at the im-rGO/GPE. (B) The graph for the relationship between $\mathrm{pH}$ and peak current; the inset shows the relationship between the peak potential and $\mathrm{pH}$ of the sensing medium.

that two protons and two electrons are involved in the electrochemical process of dopamine.

\subsection{Optimization of SWV}

Various parameters of square wave voltammetry were optimized to enhance the sensitivity of the im-rGO/GPE for dopamine. First, the amplitude of the square wave voltammetry was optimized from $0.02 \mathrm{~V}$ to $0.1 \mathrm{~V}$. The amplitude has shown a great impact on the oxidation peak current strength, and the optimum amplitude was found at $0.08 \mathrm{~V}$ (Fig. 7A). Then, the frequency was scanned from 20 to $70 \mathrm{~Hz}$, and the highest response was obtained at $50 \mathrm{~Hz}$ (Fig. 7B). The im-rGO/GPE has shown greater adsorption capability for dopamine, and it was cleared from Fig. 5 and 7C. The current was substantially enhanced as the adsorption time increased. A sharp increase in the peak signal was observed from 0 to $120 \mathrm{~s}$, and after this, it almost became constant. The constant current behavior indicated that the surface became saturated with dopamine with no need for further exploration of adsorption time. The sharp current increase just after a few seconds of adsorption time was an indication that the electrode strongly showed an affinity for dopamine adsorption.

\subsection{Calibration curve and detection limit}

Fig. 8 shows SWV for various concentrations of dopamine on the modified surface of the GPE in $0.1 \mathrm{M}$ PBS ( $\mathrm{pH}$ 6.5). Under the optimum conditions of SWV, a linear relation was observed between dopamine concentration and the peak current. A satisfactory linear relation has been observed for dopamine from 0.4 to $30 \mu \mathrm{M}$. A linear regression constant of about 0.998 was achieved. The limit of detection calculated by $\mathrm{S} / \mathrm{N}=3$ was $0.095 \mu \mathrm{M}$. The developed electrode was compared with other graphene-modified electrodes that were previously used for the detection of dopamine (Table 1).

\subsection{Comparison of the developed sensors with reported graphene-modified sensors}

From Table 1, it is clear that reduced graphene oxide is not new in the sensing of dopamine. It is always being explored in different ways to enhance the limit of quantification and detection. Few methods have been reported in which the graphene was only used for the sensing of dopamine. Y. Wang fabricated GR-CS/GCE by using the casting method and achieved the limit of quantification of $5 \mu \mathrm{M} .{ }^{32}$ Similarly, graphene was cast on a GCE, and a LOD
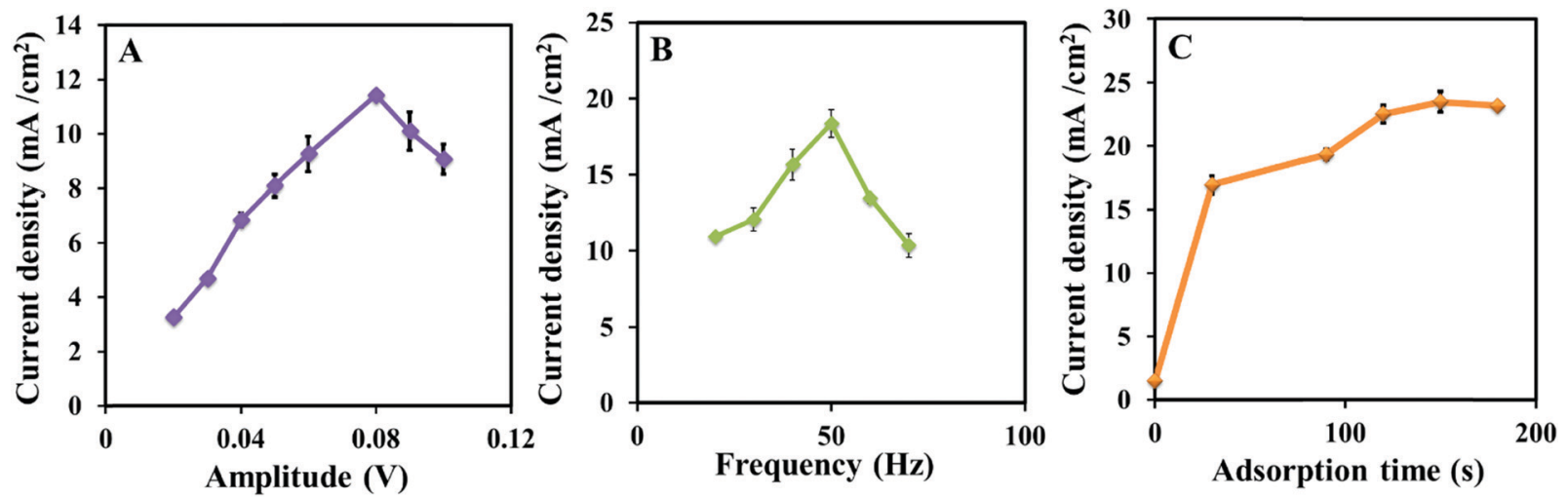

Fig. 7 Plots of the oxidation peak current vs. amplitude (A), frequency (B), at $30 \mathrm{~s}$ adsorption time, and adsorption time (C) obtained from the square wave voltammograms collected from $20 \mu \mathrm{M}$ dopamine in a PBS buffer $(0.1 \mathrm{M}, 6.5 \mathrm{pH})$. 


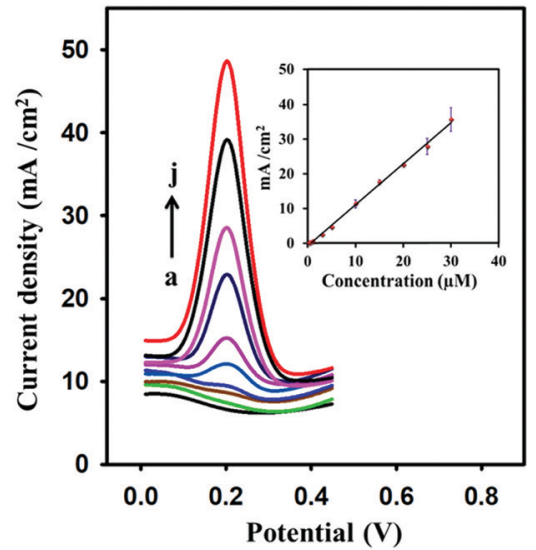

Fig. 8 Square wave voltammograms at various concentrations of dopamine: (a) $0 \mu \mathrm{M}$, (b) $0.4 \mu \mathrm{M}$, (c) $0.5 \mu \mathrm{M}$, (d) $1 \mu \mathrm{M}$, (e) $3 \mu \mathrm{M}$, (f) $5 \mu \mathrm{M}$, (g) $10 \mu \mathrm{M}$, (h) $15 \mu \mathrm{M}$, (i) $25 \mu \mathrm{M}$, and (j) $30 \mu \mathrm{M}$. The inset shows the linear relationship between oxidation peak current $(\mu \mathrm{A})$ and the concentration $(\mu \mathrm{M})$ of dopamine $\left(R^{2}=0.998\right)$.

Table 1 Comparison of the developed electrode with previously reported graphene and graphene composite electrodes

\begin{tabular}{lllll}
\hline Electrode & Technique & LOQ $(\mu \mathrm{M})$ & LOD $(\mu \mathrm{M})$ & Ref. \\
\hline GR-CS/GCE & DPV & 5 & & 32 \\
GR-GCE & DPV & 4 & 2.64 & 30 \\
GR-modified GCE & CV & 2.50 & 0.5 & 31 \\
GR-SnO $/$ /CILE & DPV & 0.5 & 0.13 & 37 \\
PPy/graphene/GCE & CV & 0.5 & 0.1 & 35 \\
GNS/paste electrode & DPV & 4 & 0.6 & 33 \\
PAM/rGO/GCE & CV & 0.3 & 0.1 & 38 \\
GR-AuNPs-CD-CS & DPV & 0.1 & 0.08 & 53 \\
modified GCE & & & & \\
PGR/GCE & Amperometry & 5 & 2 & 54 \\
3D-GO/GCE & DPV & 5 & 0.17 & 34 \\
Pt/RGO/GCE & DPV & 10 & 0.25 & 55 \\
MgO/graphene/ & DPV & 0.1 & 0.15 & 40 \\
tantalum & & & & \\
GR/Au/GR/Au/GPE & DPV & 0.1 & 0.024 & 56 \\
mp-GR/GCE & DPV & 4 & 0.15 & 41 \\
Au/Gr-AuAg & SWV & 0.3 & 0.205 & 36 \\
Au/Gr-Au & SWV & 100 & 30.3 & 36 \\
PoPD/E-RGO/GCE & DPV & 10 & 7.5 & 57 \\
im-rGO/GPE & SWV & 0.4 & 0.095 & This \\
& & & & work \\
& & & &
\end{tabular}

of $4 \mu \mathrm{M}$ was obtained. ${ }^{30} \mathrm{~A}$ similar limit of quantification of $5 \mu \mathrm{M}$ was achieved by using pristine graphene (PGR/GCE) ${ }^{54}$ and 3D reduced graphene oxide (3D-rGO/GCE). ${ }^{34}$ These electrodes were prepared by the casting method. ${ }^{34,54}$ In another method, a graphene-modified GCE was used, and the LOQ attained was $2.5 \mu \mathrm{M}^{31}$ The multi nanopore graphene-modified GCE has shown a LOQ of $4 \mu \mathrm{M}^{41}$ All these electrodes have used just graphene for modification. In comparison with these electrodes, the electrode developed in this work is more sensitive, with the limit of quantification of $0.4 \mu \mathrm{M}$. The LOQ and the LOD of the im-rGO/GPE were even better compared to many graphene composite electrodes (Table 1). The im-rGO/GPE modification process is simple and achieved in a short time compared to the casting and other multistep methods. The improvement of LOQ and the LOD of the GPE might be due to the effective reduction of graphene oxide in the presence of the ionic medium. Better control of the thickness of the reduced graphene oxide layer can be achieved by directly reducing graphene oxide on the surface of the GPE. The reusability of the modified electrodes can be affected strongly by the electro-polymerization of the dopamine and production of byproducts during electrochemical sensing of dopamine on the electrode surface. The im-rGO/GPE copes with this challenge by producing a new surface to replace the old one in a short time. The electrode is cheap, and the modification material is cost-effective, which provides the opportunity to develop disposable electrodes for dopamine sensing.

\subsection{Detection of dopamine in real samples and interferences study}

The dopamine in a real sample was analyzed with the developed im-rGO/GPE sensor. A healthy person's urine sample was collected from a volunteer and diluted 200 times with $0.1 \mathrm{M}$ PBS buffer. Various concentrations of dopamine were spiked $(5,10,15 \mu \mathrm{M})$ in the urine sample and the im-rGO/GPE was used to measure the response of the oxidation peak current of the dopamine. The recoveries were found in the range of 96 to $107 \%$ (Table S1, ESI $\dagger$ ). The good recoveries indicated that the developed electrode could be applied for real samples. The major interfering species in the determination of dopamine are uric acid and ascorbic acid. A well-separated peak of dopamine was observed in the presence of uric acid and ascorbic acid. A $10 \%$ variation in current was observed for $15 \mu \mathrm{M}$ dopamine in the presence of $1 \mathrm{mM}$ ascorbic acid. Moreover, a weak response of the ascorbic acid was observed at $0.046 \mathrm{~V}$, which further endorses the rGO-GPE as more specific towards dopamine in the presence of ascorbic acid (Fig. 9). This could be due to a few negative carboxylate ions still being present after the im-rGO on the GPE surface, which reduces the adsorption capability of the ascorbic acid. Moreover, the optimized parameters of the SWV are more sensitive and selective for dopamine. The high concentration of other biomolecules like $\mathrm{L}$-methionine, adenine, glucose, l-tryptophan, and presence of $\mathrm{K}^{+}, \mathrm{Na}^{+}, \mathrm{SO}_{4}{ }^{-2}$, and $\mathrm{Cl}^{-1}$ ions has shown current variations from \pm 1.85 to $\pm 8.83 \%$.

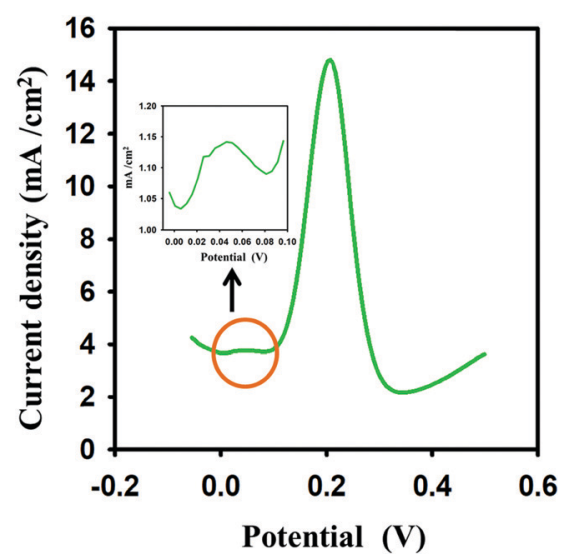

Fig. 9 Square wave voltammetric response of $15 \mu \mathrm{M}$ dopamine in the presence of $1 \mathrm{mM}$ ascorbic acid. Inset showing the ascorbic acid peak current. 


\subsection{Developed method reproducibility}

The common problem associated with the electrodes is the surface fouling after the first measurement of dopamine. The same modified surface is reused in most of the reported electrodes, and many methods are adopted to clean the surface from byproducts before the second measurement. During the cleaning process, the modified surface can be damaged, and some of the byproducts still can reside on the surface. This method is generally adopted if the modification process is multistep, time-consuming, or the modifying materials are expensive. In our case, the electrode is disposable, and after each measurement, the new modified surface was prepared effortlessly in a short time using ionic medium supported GO. The renewability of the surface makes it foul free for the dopamine measurement. The RSD obtained in six repeating ASSWV was $4.1 \%$. The electrode surface was renewed before each measurement of dopamine.

\section{Conclusions}

A disposable ionic medium reduced graphene oxide modified electrode has been developed for trace level quantification of dopamine. The electrode modification process is simple and can be completed in a short time. It provided an opportunity to produce a new electrode surface for each measurement. The reduced graphene oxide produced on the GPE surface from ionic medium supported graphene oxide was found to be more effective compared to the reduced graphene oxide obtained from the graphene oxide dispersed in a water medium. The im-rGO/GPE has shown greater electroactive surface area compare to w-rGO/GPE under the same set of conditions. The im-rGO on the GPE has substantially improved the electroactive surface area of GPE from 0.063 to $0.631 \mathrm{~cm}^{2}$ for dopamine. The im-rGO layer on the GPE has shown a good affinity towards dopamine that might be due to the presence of the residual oxygen-containing functionalities after reduction. A linear relationship was observed for oxidation peak current and concentration of dopamine from 0.4 to $30 \mu \mathrm{M}\left(R^{2}=0.998\right)$. The limit of detection was found to be about $0.095 \mu \mathrm{M}(\mathrm{S} / \mathrm{N}=3)$ with im-rGO/GPE. The developed sensor has displayed good reproducibility with an RSD of $4.1 \%(n=6)$. The developed sensor can be proven as an excellent single-use electrode for the cost-effective sensing of dopamine.

\section{Conflicts of interest}

There are no conflicts to declare.

\section{Acknowledgements}

The authors extend their appreciation to the Deputyship for Research \& Innovation, Ministry of Education in Saudi Arabia, for funding this research work through the project number RDO2019-002-IRMC.

\section{References}

1 J. Wang and A.-N. Kawde, Anal. Chim. Acta, 2001, 431, 219-224.

2 E. Alipour, M. R. Majidi, A. Saadatirad, S. M. Golabi and A. M. Alizadeh, Electrochim. Acta, 2013, 91, 36-42.

3 K. Aoki, T. Okamoto, H. Kaneko, K. Nozaki and A. Negishi, J. Electroanal. Chem., 1989, 263, 323-331.

4 A. M. Bond, P. J. Mahon, J. Schiewe and V. Vicente-Beckett, Anal. Chim. Acta, 1997, 345, 67-74.

5 P. Annu, S. Sharma, R. Jain and A. N. Raja, J. Electrochem. Soc., 2020, 167, 037501.

6 J. I. Gowda, G. S. Hurakadli and S. T. Nandibewoor, Anal. Chem. Lett., 2017, 7, 389-401.

7 I. G. David, D.-E. Popa and M. Buleandra, J. Anal. Methods Chem., 2017, 2017, 1-22.

8 A.-N. Kawde, N. Baig and M. Sajid, RSC Adv., 2016, 6, 91325-91340.

9 A. Rana and A.-N. Kawde, J. Chin. Chem. Soc., 2016, 63, 668-676.

10 M. Ozsoz, A. Erdem, K. Kerman, D. Ozkan, B. Tugrul, N. Topcuoglu, H. Ekren and M. Taylan, Anal. Chem., 2003, 75, 2181-2187.

11 H. Karadeniz, A. Erdem, A. Caliskan, C. M. Pereira, E. M. Pereira and J. A. Ribeiro, Electrochem. Commun., 2007, 9, 2167-2173.

12 S. Ayaz, S. Karakaya, G. Emir, D. G. Dilgin and Y. Dilgin, Microchem. J., 2020, 154, 104586.

13 M. Muti, F. Kuralay and A. Erdem, Colloids Surf., B, 2012, 91, 77-83.

14 A. Sreekumar, P. Navaneeth, P. V. Suneesh, B. G. Nair and T. G. S. Babu, Microchim. Acta, 2020, 187, 113.

15 A.-N. Kawde, M. A. Aziz, M. El-Zohri, N. Baig and N. Odewunmi, Electroanalysis, 2017, 29, 1214-1221.

16 W. Zhao, B. Ni, Q. Yuan, Y. Wang, Q. Zhang and X. Wang, Langmuir, 2017, 33, 8070-8075.

17 A. F. Khan, D. A. C. Brownson, E. P. Randviir, G. C. Smith and C. E. Banks, Anal. Chem., 2016, 88, 9729-9737.

18 X. Guo, H. Yue, S. Huang, X. Gao, H. Chen, P. Wu, T. Zhang and Z. Wang, Microchim. Acta, 2020, 187, 218.

19 A. Ejaz, Y. Joo and S. Jeon, Sens. Actuators, B, 2017, 240, 297-307. 20 Y. Yao, J. Zhong, Z. Lu, X. Liu, Y. Wang, T. Liu, P. Zou, X. Dai, X. Wang, F. Ding, C. Zhou, Q. Zhao and H. Rao, Microchim. Acta, 2019, 186, 795.

21 H. Yang, D. Liu, X. Zhao, J.-H. Yang, H. Chang, R. Xing and S. Liu, Anal. Methods, 2017, 9, 3191-3199.

22 M. Pumera, Chem. Soc. Rev., 2010, 39, 4146-4157.

23 Y. Liu, X. Dong and P. Chen, Chem. Soc. Rev., 2012, 41, 2283-2307.

24 Y. Shao, J. Wang, H. Wu, J. Liu, I. A. Aksay and Y. Lin, Electroanalysis, 2010, 22, 1027-1036.

25 M. Pumera, Mater. Today, 2011, 14, 308-315.

26 S. Wu, Q. He, C. Tan, Y. Wang and H. Zhang, Small, 2013, 9, 1160-1172.

27 N. Baig and A. Kawde, Anal. Methods, 2015, 7, 9535-9541.

28 R. Zhang and W. Chen, Biosens. Bioelectron., 2017, 89, 249-268. 
29 Y. Bai, T. Xu and X. Zhang, Micromachines, 2020, 11, 60.

30 Y.-R. Kim, S. Bong, Y.-J. Kang, Y. Yang, R. K. Mahajan, J. S. Kim and H. Kim, Biosens. Bioelectron., 2010, 25, 2366-2369.

31 X. Ma, M. Chao and Z. Wang, Anal. Methods, 2012, 4, 1687-1692.

32 Y. Wang, Y. Li, L. Tang, J. Lu and J. Li, Electrochem. Commun., 2009, 11, 889-892.

33 S.-Q. Liu, W.-H. Sun and F.-T. Hu, Sens. Actuators, B, 2012, 173, 497-504.

34 B. Yu, D. Kuang, S. Liu, C. Liu and T. Zhang, Sens. Actuators, $B, 2014,205,120-126$.

35 Z. Zhuang, J. Li, R. Xu and D. Xiao, Int. J. Electrochem. Sci., 2011, 6, 2149-2161.

36 S. Pruneanu, A. R. Biris, F. Pogacean, C. Socaci, M. Coros, M. C. Rosu, F. Watanabe and A. S. Biris, Electrochim. Acta, 2015, 154, 197-204.

37 W. Sun, X. Wang, Y. Wang, X. Ju, L. Xu, G. Li and Z. Sun, Electrochim. Acta, 2013, 87, 317-322.

38 Y. J. Yang, Electrochim. Acta, 2014, 146, 23-29.

39 L. Fritea, A. Le Goff, J.-L. Putaux, M. Tertis, C. Cristea, R. Săndulescu and S. Cosnier, Electrochim. Acta, 2015, 178, 108-112.

40 L. Zhao, H. Li, S. Gao, M. Li, S. Xu, C. Li, W. Guo, C. Qu and B. Yang, Electrochim. Acta, 2015, 168, 191-198.

41 X. Zhu, Y. Liang, X. Zuo, R. Hu, X. Xiao and J. Nan, Electrochim. Acta, 2014, 143, 366-373.

42 W. S. Hummers and R. E. Offeman, J. Am. Chem. Soc., 1958, 80, 1339.

43 F. Y. Ban, S. R. Majid, N. M. Huang and H. N. Lim, Int. J. Electrochem. Sci., 2012, 7, 4345-4351.
44 Z. Li, W. Zhang, J. Guo, B. Yang and J. Yuan, Vacuum, 2015, 117, 35-39.

45 J. Kudr, L. Richtera, L. Nejdl, K. Xhaxhiu, P. Vitek, B. Rutkay-Nedecky, D. Hynek, P. Kopel, V. Adam and R. Kizek, Materials, 2016, 9, 31.

46 P. Rattanarat, A. Suea-Ngam, N. Ruecha, W. Siangproh, C. S. Henry, M. Srisa-Art and O. Chailapakul, Anal. Chim. Acta, 2016, 925, 51-60.

47 E. Colín, S. C. Avendaño, M. T. Ramírez, M. Romero-Romo and M. Palomar-Pardave, Int. J. Electrochem. Sci., 2012, 7, 6097-6105.

48 W. Li and Y. J. Yang, J. Solid State Electrochem., 2014, 18, 1621-1626.

49 T. Ndlovu, O. A. Arotiba, S. Sampath, R. W. Krause and B. B. Mamba, Int. J. Electrochem. Sci., 2012, 7, 9441-9453.

50 G. Hernández-Cancel, D. Suazo-Dávila, J. Medina-Guzmán, M. Rosado-González, L. M. Díaz-Vázquez and K. Griebenow, Anal. Chim. Acta, 2015, 854, 129-139.

51 W. Zhu, T. Chen, X. Ma, H. Ma and S. Chen, Colloids Surf., B, 2013, 111, 321-326.

52 L. N. Qu, J. Wu, X. Y. Sun, M. Y. Xi and W. Sun, J. Chin. Chem. Soc., 2010, 57, 701-707.

53 J. Tang, L. Zhang, Y. Liu, J. Zhou, G. Han and W. Tang, Electroanalysis, 2014, 26, 2057-2064.

54 S. Qi, B. Zhao, H. Tang and X. Jiang, Electrochim. Acta, 2015, 161, 395-402.

55 T.-Q. Xu, Q.-L. Zhang, J.-N. Zheng, Z.-Y. Lv, J. Wei, A.-J. Wang and J.-J. Feng, Electrochim. Acta, 2014, 115, 109-115.

56 N. Baig and A.-N. Kawde, RSC Adv., 2016, 6, 80756-80765.

57 X. Liu, H. Zhu and X. Yang, RSC Adv., 2014, 4, 3743-3749. 